\section{Rheology at Gothenburg}

from $D . R$. Oliver

The Seventh International Congress in Rheology was held at Chalmers University of Technology, Gothenburg, Sweden on August 23-27, 1976, organised by Professor Josef Kobat and colleagues (Chalmers University).

RHEOLOGY is the study of the deformation and flow of materials, but a visit to Gothenburg provided opportunities to observe the deformation and flow of travellers cheques! The subject subdivisions chosen by the organisers were polymer solids, polymer melts, fluids, polymer solutions, theory, suspensions, biorheology, methods, metals, concrete and food. This order represents a ranking in terms of numbers of papers presented: new subjects such as biorheology (13 papers) and food (4) seem to be making little impact on the combined polymer topics (149). The apparent fall in the number of theoretical papers (23) is illusory, since papers under other headings contain extensive theoretical work and one new concept in particular seems to be gaining momentum, the search for rheological equations of state consistent with thermodynamics (J. G. Oldroyd (University of Liverpool) and G. Astarita (University of Naples)). Another link between rheology and physics was provided in a historical lecture by $\mathrm{H}$. Markovitz (CarnegieMellon University, Pittsburgh) who pointed out that $100 \mathrm{yr}$ ago Boltzmann formulated his principle of superposition, according to which stresses present at a given time depend not only on the deformation at the time but also on previous deformations, the influence of which decreases with increasing elapsed time. The suggestion was made that Boltzmann's ideas should be given status comparable with those of Maxwell in the early development of theories of viscoelastic behaviour.

Flow visualisation is widely used in rheology: some unusual and beautiful extrudate flow forms were shown by N. Bergam (CIIR, Oslo) and die-entry flows, with customary flair, by Professor Giesekus (University of Dortmund). The quadruple vortex formed in a Taylor 4-roll mill was demonstrated by J. M. Broadbent (University of Wales, Aberystwyth) and the flow pattern around a sphere moving in a viscoelastic liquid by D. Sigli and M. Coutanceau (University of Poitiers). The pattern of a recoiling flow upstream of an orifice upon sudden cessation of flow was shown by R. J. Gordon (University of Florida) and a remarkable tri-modal Weissenberg effect induced by torsional oscillation of a rod in a viscoelastic liquid by D. D. Joseph (University of Minnesota). The liquid climbing the rod builds up into three (or even four) distinct lobes before eventually collapsing.

A computational method finding increasing acceptance is the finite element system, in which the uniform grid of a finite difference network is replaced by a set of irregular elements which concentrate attention around areas of particular interest. R. Tanner (University of Sydney) obtained valuable information regarding velocity profiles at the exit end of a capillary tube and was able to predict jet swell for Newtonian fluids; N. Davids (Pennsylvania State University) and M. L. Wenner (General Motors, Michigan) used finite elements for the study of uniaxial waves in a viscoelastic rod; D. S. Malkus (National Bureau of Standards, Washington) calculated hole pressure error, and G. Geymonat (CNRS, Marseilles) and M. Raous (Poletecnico, Turin) applied the technique to the near-solid problems of the behaviour of turbine blades and to time-dependent effects in ageing concrete. The finite element method will become widely used in rheology and other branches of fluid flow.

The flow of blood was the dominant theme of the biorheology section. New viscosity concentration relationships were obtained by D. Quemada (University of Paris) and hysteresis effects in whole human blood in Couette flow studied by A. Apelblat et al. (Paris). These experiments showed that the aggregation of red cells is both a timeand shear-dependent phenomenon producing hysteresis loops in rotary instruments. The possible migration of red cells away from the walls should not be ignored; these effects occur strongly in flow down fine capillaries and produce the Fahraeus-Lindquist effect, that is, lower than normal apparent blood viscosity. M. Singh (IST, Madras) and N. A. Coulter (University of North Carolina) studied the latter behaviour in oscillatory flow down capillaries and reached the interesting conclusion that the effect begins at larger tube radii than for steady flowthis may help the human body considerably! In a significant paper $\mathrm{L}$. E. Gelin (University of Gothenburg) showed how the rheological changes in blood following shock or trauma influence its distribution in fine capillaries. A Perspex model allowed blood to flow along capillaries and then to choose a "straight on" or "branching" path. For highly aggregated blood (such as that following shock), the forward flow contained more red cells and the side flow less, particularly for very narrow tubes. In critically ill patients, this may lead to the total immobilisation of red cells, visible as "red shock".

Several new concepts were introduced. B. Mena and O. Manero (National University of Mexico) showed that the flow rate of a viscoelastic liquid along a tube could be increased 10 -fold by the low frequency axial oscillation of the tube, producing spectacular spurts of liquid. A paper by $\mathrm{K}$. Walters (University of Wales, Aberystwyth) described his "torsional balance" variant of the rheogoniometer, which started life as a squeeze film system but has become a potential rival to jet thrust as a high-shear steady-state normal stress measuring instrument.

Extensional measurements continue. L. Nicolais (University of Naples) and colleagues showed that the extensional viscosity of polymer relations containing fine glass beads in suspension is actually reduced due to the presence of the filler; conversely D. R. Oliver and R. C. Ashton (University of Birmingham) showed that the polymers added to car lubricating oils cause large increases of extensional viscosity in both uniaxial and biaxial flows.

A novel fibre foam was described by S. Turner and F. N. Cogswell (ICI, Welwyn Garden City). This fibre/resin/ air matrix is easily bonded to other materials and is light and strong. The uses suggested include shock absorbent packing, fenders and riot shields-certainly a topical development.

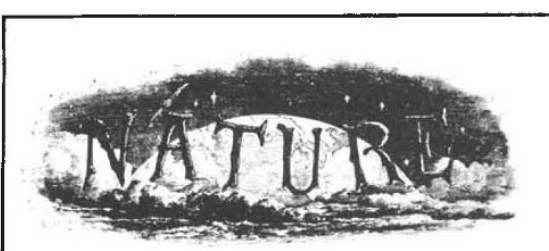

\section{A hundred years ago}

THE Fellows of the College of Physicians of Dublin have deliberately determined to admit Miss Edith Pechey to the examination for the L.K.Q.C.P.I., and have thus thrown open the portals of the medical profession to all comers, whether they be "persons" of the male or female sex. However pregnant of results this decision may be, says the Medical Press and Circular, it does not seem to us that any other conclusion was possible, and we expect to see a similar ingress allowed to the ladies by all other bodies. The Queen's University, it is anticipated, will be the next to follow suit, and these fortresses having surrendered at discretion, it is impossible that others can long sustain the seige.

From Nature, 14, October 19, 560; 1876. 